\title{
Proceedings
}

\section{Prediction of Tree Age Distribution Based on Survival Analysis in Natural Forests: A Case Study of Preserved Permanent Plots in the University of Tokyo Hokkaido Forest, Northern Japan}

\author{
Pavithra Rangani Wijenayake ${ }^{1}$ Takuya Hiroshima 2,* \\ 1 Department of Forest Science, Graduate School of Agricultural and Life Sciences, The University of Tokyo, \\ 1-1-1 Yayoi, Bunkyo-ku, Tokyo, 113-8657, Japan; rangani@g.ecc.u-tokyo.ac.jp \\ 2 The University of Tokyo Hokkaido Forest, Graduate School of Agricultural and Life Sciences, The \\ University of Tokyo, 9-61, Furano, Hokkaido, 079-1563, Japan; hiroshim@uf.a.u-tokyo.ac.jp \\ * Correspondence:rangani@g.ecc.u-tokyo.ac.jp
}

\begin{abstract}
In forests, tree mortality is strongly determined by complex interactions between multiple biotic and abiotic factors, and analysis of tree mortality is widely implemented in forest management. However, age-based tree mortality remains poorly evaluated quantitatively at the stand scale of the uneven-aged forests. The objective of this study is to predict the age distribution of living and dead trees based on survival analyses. We used a combination of tree-ring and census data from the two preserved permanent plots in the University of Tokyo Hokkaido Forest in panmixed and sub-boreal natural forests, Hokkaido, northern Japan, to derive site-specific survival models. All the living trees (diameter at breast height $\geq 5 \mathrm{~cm}$ in 2009) were targeted to identify tree ages using a RESISTOGRAPH, a semi non-destructive device. Periodical tree age data with a 10year age class were used during the observation periods of 2009-2019, and all the changes (i.e., death and new in-growth) during the periods were recorded. We found the time stabilities of survival functions between periods in advance. The results showed that the parametric survival analysis with Weibull distribution successfully yielded the mortality rate, mortality probability, and survival probability in each plot. Finally, we predicted the future age class distribution of living and dead trees of each plot based on the survival analyses results and discussed its management implications. We recommended that the estimated mean lifetime was facilitated to make decisions on the selection of harvesting trees in the uneven forest management based on selective cutting.
\end{abstract}

Keywords: natural forest; survival analysis; tree age distribution

\section{Introduction}

Changes in survival probability of forests will have environmental [1] and severe economic consequences. Survival probability changes can influence on forest management decisions $[2,3]$. There is a strong need for long-term tree mortality studies [4-7] considering the threat of global climate change, which is forecasted to increase tree mortality in forests worldwide [8].

Because of the difficulty in detecting the exact age of trees, the researchers mainly used DBH, dominant height, basal area, growth rate, or competition index by avoiding age-based measures to develop mortality probabilities. Generally, a tree's full survival time is unknown; unlike in logistic regressions, the right censoring and left truncation approaches of survival analysis can be applied to handle these data. For the first time, [9] suggested survival analysis techniques to solve forest mortalities, even though these applications were limited to even-aged forest stands [10,11]. Woodall [12] carried out a survival analysis based on Diameter at Breast Height (DBH). Finally, he emphasized the importance of exact age on survival analysis of forests. 
The principles of the approach presented in this paper are related to the basic idea shown in Hiroshima [13], who applied more flexible techniques of survival analysis based on non-parametric and parametric approaches. This method is based on annual tree ring measurements by a seminondestructive device as well as periodic inventory data of secondary natural forests in Japan. However, except for this, studies focusing on age-based survival analysis on uneven-aged natural forests are comparatively rare because they require special methods to detect trees age and long-term and large-scale observations as well. In this study, we use a particular device to detect tree age and take advantage of the long-term research site in the preserved area, located in the University of Tokyo Hokkaido forest (UTHF), profoundly affected by wind-induced forest mortality. Overall, the purpose of this study was to perform a survival analysis of individual tree populations in natural forest stands of the UTHF, Japan, to predict the age distribution of living and dead trees. First, we applied survival analysis techniques to each plot to find out non-parametric estimations. Secondly, parametric Weibull estimates were developed to predict future age class distributions. Specifically, we showed how the stand age estimations differ with mean lifetime after ingrowth in each plot.

\section{Materials and Methods}

\subsection{Study Area}

The study was carried in the UTHF, which is located at Furano (central Hokkaido, northern Japan).

In this study, we chose two permanent plots ( 0.25 ha each), which are located at an elevation of 681 and $580 \mathrm{~m}$ in the preserved area (Figure 1). This middle elevation represents the range of species composition as well as stand structure with wind-damaged history. The typical vegetation types of these plots: a coniferous and broad-leaved mixed forest dominated by Abies sachalinensis and Acer species.

\subsection{Collection of tree age data}

Across two plots, we detected the number of tree rings at breast height $(1.3 \mathrm{~m})$ of 451 trees in 2019 using a semi non-destructive device called the RESISTOGRAPH developed by [14]. The 10-year observation period was set as 2009-2019. All the target trees were alive and had DBH $\geq 5 \mathrm{~cm}$ in 2009, and some of these trees were died by 2019. The status of live/dead and new in-growth trees was counted at the end of each observation period. These field measurements data of tree rings in 2019 were extracted via DECOM software to annul tree ring detection. Figure 2 describes the annual rings detected in one of the alive Abies trees using the DECOM software. RESOSTOGRAPH measurements were ineffective for the badly rotten and center decayed trees. Cross-sectional wood discs were cut at the field to count the annual rings. We manually counted the annual rings for those species and estimated the regression equation between the radius of the wood disk $(x)$ and the number of annual rings $(y)$ [15]. The age after in-growth was determined by inputting the radius data into simple threedimensional equations fitted to a scatter diagram. This method was followed by $25 \%$ of the sample trees.

We analyzed/estimated 237 and 214 trees of plot number 5225 and 5240, respectively. The relevant age at the beginning of the observation periods in 2009 was decided by deducting ten years from the resulted tree age in 2019 of each tree. Finally, tree ages were classified as ten years per one age class. 

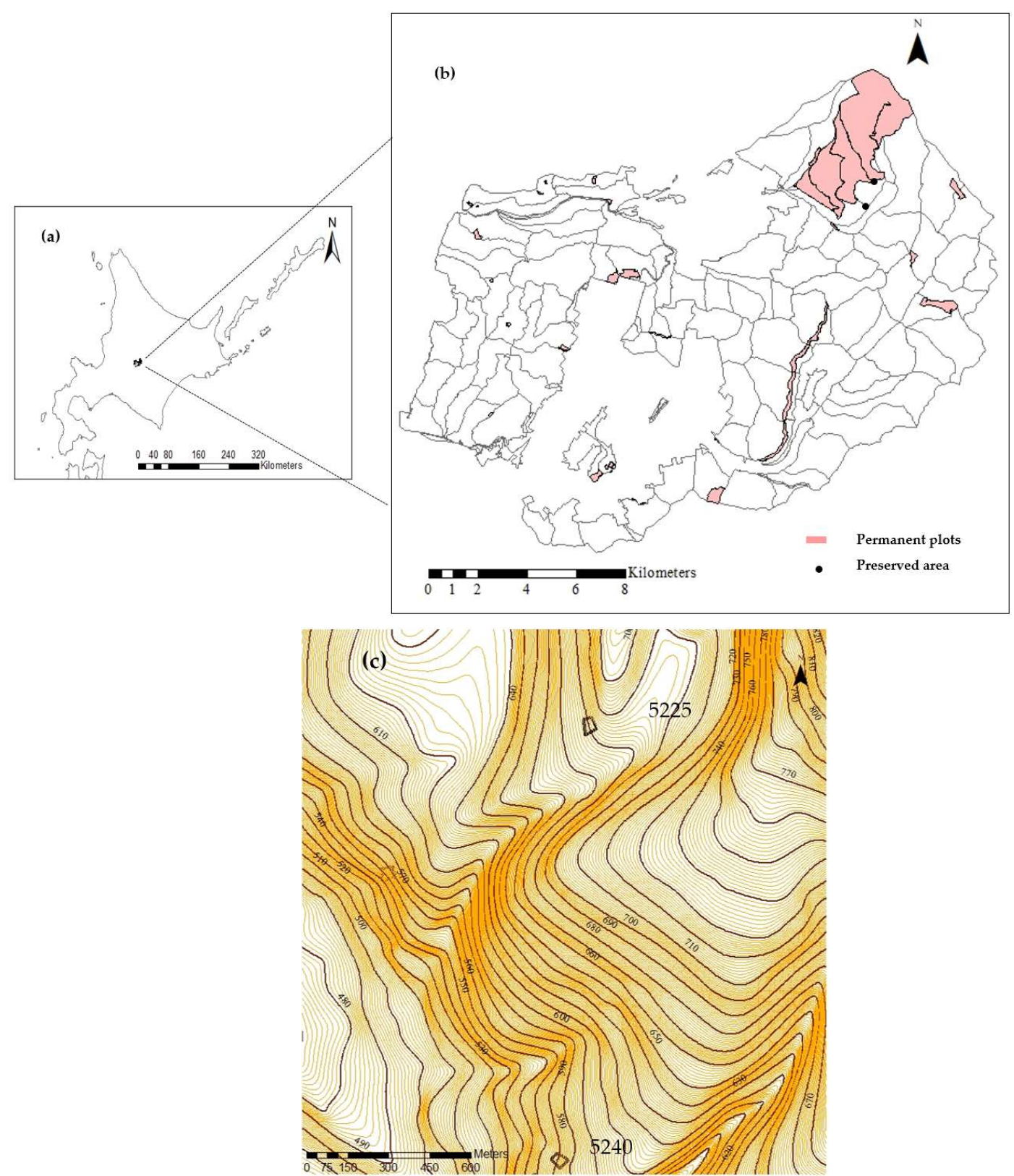

Figure 1. Maps showing the locations of study area and plots. (a) UTHF of the Hokkaido island of Japan, (b) preserved area and permanent plots of the UTHF, (c) Contour map of the two preserved permanent plots of UTHF.

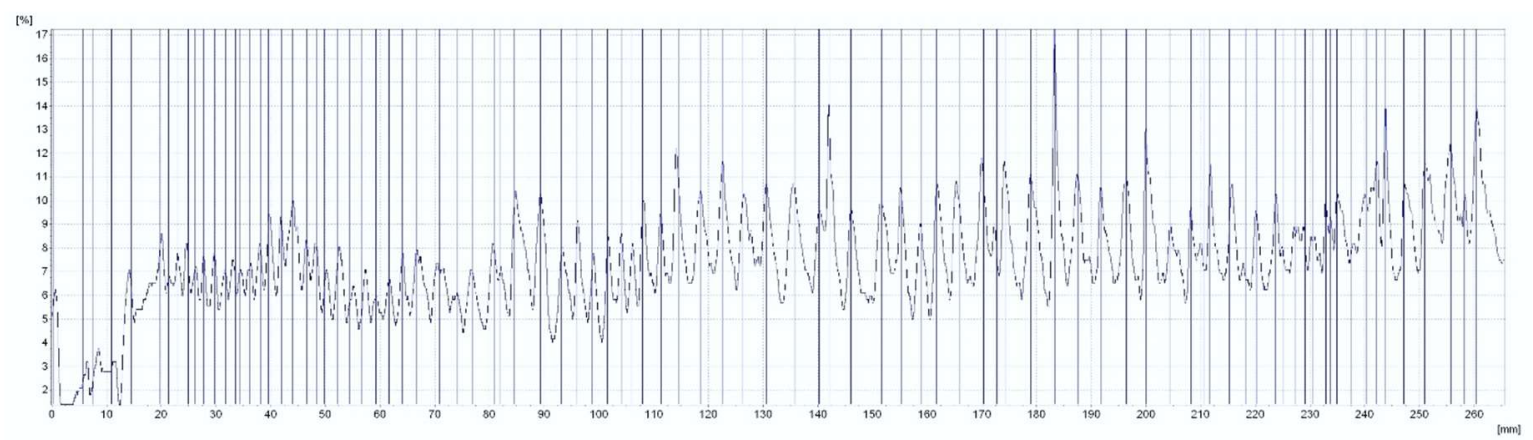

Distance from bark to pith

Figure 2. Manually detected annual rings of Abies sachalinensis tree of plot 5240 which is after detecting from the DECOM software. 


\subsection{Survival Analysis of Target Trees}

The survival probability functions are the essential elements of this analysis, as it reflects the overall performance of two plots. These functions were developed based on previous studies [1618]. The survival data demonstrated that there were both dead and alive trees in the study plots at the end of the observation period. In these instances, the survival time is described as both "censored" and "truncated." Right censoring happens when a target leaves the study before an event occurs, or the follow-up ends before it happens. On the other hand, left truncation happens when an object is not observed from the start of the study but instead enters the observation period in this study later.

The Kaplan-Meier survival estimator is an important tool for analyzing censored data, and the Kaplan-Meier curve [19] can be used to estimate the distribution of tree mortality.

The survival analysis was performed to describe survival probability distribution by following the above studies. The survival function of Kaplan Meier (KM) estimate is as follows:

$$
\hat{r_{t}}=\prod_{k<t}\left(1-\hat{p}_{k}\right)=\prod_{k<t}\left(1-\frac{d_{k}}{a_{k}+d_{k}}\right) .
$$

Where $\hat{r}_{t}$ represents the survival function and $d_{k} \backslash\left(a_{k}+d_{k}\right)$ represents the hazard function at the $t^{\text {th }}$ age class.

Besides non-parametric analysis, Weibull distribution was applied to predict the future age distribution of living and dead trees. This parametric analysis estimates the parameters of $m$ and $k$ of the Weibull distribution to check the fitness of these distributions for Kaplan-Meier estimates.

$$
f(t ; m, k)=\frac{k}{m^{k}} t^{k-1} e^{-\left(\frac{t}{m}\right)^{k}}
$$

Equation numbers 3 and 4 were used to predict the living and dead tree distribution after the 2nd age class of each plot.

$$
\begin{gathered}
b_{t}=\left(a_{t-1}+d_{t-1}\right)\left(1-p_{t-1}\right), t \geq 2 \\
c_{t}=\left(a_{t-1}+d_{t-1}\right)\left(p_{t-1}\right), t \geq 2
\end{gathered}
$$

where $b_{t}$ and $c_{t}$ are the number of living trees and dead trees in the current period by age class $t$ based on the number of living trees $a_{t}$, dead trees $d_{t}$ and corresponding tree mortality rate $p_{t}$ derived from Weibull analysis for the previous period.

\section{Results}

The species composition of living and dead trees of two plots were different, so we avoided combining the tree data in the two plots to carry out the following survival analyses. We found these Kaplan Meier curves were time stable in both plots as a result of the log-rank test [15]; therefore, these survival probabilities were used to carry out the future predictions in the following parametric analyses.

The Weibull distribution was applied to smooth the stepwise values of non-parametric analysis for prediction purposes. Table 1 shows the estimated parameters of $m$ and $k$ and the mean and standard deviation of the Weibull distributions of 2 plots. The higher mean and standard deviation values were found in plot 5225. The mean value represents the mean lifetime after in growth, and according to the Weibull estimates, the mean lifetime of 5225 and 5240 is 137 and 95 years, respectively. 
Table 2. Probability distributions of Weibull for each plot.

\begin{tabular}{ccc}
\hline Weibull parameter & Plot 5225 & Plot 5240 \\
\hline$m$ & 13.214 & 10.271 \\
$k$ & 0.925 & 1.275 \\
Mean & 13.7 & 9.5 \\
Standard Deviation & 14.8 & 7.5 \\
\hline \multicolumn{3}{c}{$m$ = scale parameter, $k=$ shape parameter }
\end{tabular}

For validation purposes, living and dead tree distributions of each plot in the observation period were calculated based on equations 3 and 4 . The studied plots were characterized by uneven age class distribution of trees. However, the shape of the age class distribution varied between the two plots. The proportion of living and dead trees of 5225 declines gradually toward the older age classes, while 5240 represents a slight difference in the age class frequencies at younger age classes. For $t \geq 2$, the total number of Weibull calculations were 157 (plot 5225) and 159 (plot 5240), while observations were 155 (plot 5240) and 158 (plot 5240), respectively. The estimation of surviving trees ended up by $1.29 \%$ and $0.63 \%$ error ratios of 5225 and 5240 and represented the characteristics of age distributions correctly. The error ratio of the dead tree in 5225 and 5240 were $16.67 \%$ and $0 \%$, respectively. The error ratios based on parametric analysis nearly agreed with the observed data, which showed validation results were enough precise.
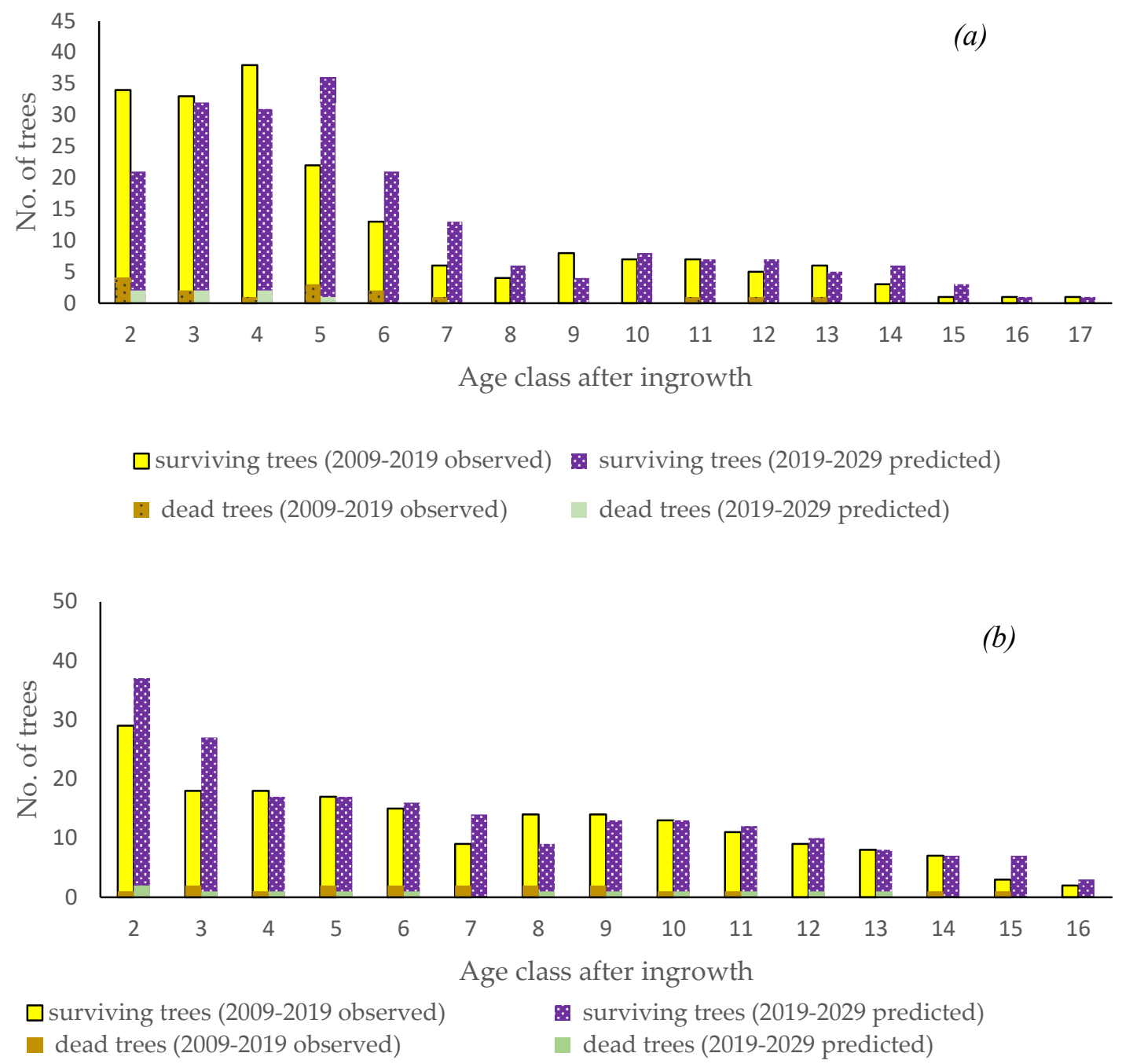

Figure 3. Future prediction of age class distribution of living and dead trees (2019-2029): (a) plot 5225; (b) plot 5240. 
Based on the estimated $p_{t}$ during 2009-2019 and assuming these mortality rates are stable over time, the age class distribution in the next period, 2019-2029, was predicted in each plot. The resulting living and dead tree distributions are shown in figure 3 . The predicted numbers of living trees are less than the 2009-2019 period, especially in the second, third, and fourth age classes. In contrast, the living tree projections of second and third age classes were higher than those observed in 5240.

\section{Discussion}

Estimates of shape parameters differed in the range of $k<1$ for 5225 and $k>1$ for 5240 , which led to the differences between the shape of curves in two plots. Because dead trees in 5240 tended to concentrate on the older age classes compared with 5225, the estimates of $k$ for 5240 showed a higher value than 5225 . These differences of shapes resulted in the difference of mean lifetime of 137 years in 5225 and 95 years in 5240, which is mainly caused by the difference in mortality rates between the plots. The older tree death of 5240 led to a lower mean lifetime rather than in 5225 and, which also represented a higher number of trees could survive in 5225 than 5240. The weighted means of these biological lifetimes are calculated as 171 and 173 years in two plots based on species and number of trees. Thus, the above mean lifetime of 137 and 95 years and also the maximum stand ages of 159 and 149 years in both plots were relatively near to the mean biological lifetime of 171 and 173 years. These facts imply the matured states of these stands, which lead to the stable states of survival probabilities over time and enable future predictions based on the estimated survival probabilities.

Forest managers can rely on these predicted age class distributions in decision-making, such as harvesting tree selection based on SSMS. The resulting future prediction of dead trees concerning age class will facilitate as a harvesting indicator prior to the death of tree. The productivity of harvested trees can be enhanced by this type of early identification of likely-to-die or decaying trees.

Thus, future predictions of the age class distribution of each plot like this study can be used as site-specific management plans to identify living and dead trees of each age class though it is essential to carry out dendrological survival analysis instead of whole stand analysis.

\section{Conclusions}

In this study, we succeeded to estimate survival probabilities both by the non-parametric and parametric way. We also showed the availability of future prediction using parametric estimates of survival probabilities. The results implied that natural forest management based on selective cutting like SSMS in UTHF could be practiced more efficiently by considering the estimated mean lifetime derived from survival analysis.

However, the survival probabilities estimated in this study should be used carefully for longterm predictions of forest dynamics because they do not include the effect of catastrophic disturbances, which can often have significant influences on forests. Therefore, in our future work, it is essential to incorporate these variables to enhance the practical applicability of survival probability models.

Author Contributions: Data analysis, writing-original draft preparation, P.R.W.; Supervision, review and editing, T.H. All authors have read and agreed to the published version of the manuscript.

Funding: This study was funded by the JSPS KAKENHI, Grant Number JP19K06142.

Acknowledgments: We thank the technical staff of the UTHF for their technical support throughout the fieldwork and their help with the tree census data.

\section{References}

1. Seidl, R.; Schelhaas, M.J.; Rammer, W.; Verkerk, P.J. Increasing forest disturbances in Europe and their impact on carbon storage. Nat. Clim. Chang. 2014, 4, 806-810, doi:10.1038/nclimate2318.

2. Griess, V.C.; Knoke, T. Bioeconomic modeling of mixed Norway spruce-European beech stands: Economic consequences of considering ecological effects. Eur. J. For. Res. 2013, 132, 551-552, doi:10.1007/s10342-0130692-3. 
3. Paul, C.; Brandl, S.; Friedrich, S.; Falk, W.; Härtl, F.; Knoke, T. Climate change and mixed forests: how do altered survival probabilities impact economically desirable species proportions of Norway spruce and European beech? Ann. For. Sci. 2019, 76, 1-15, doi:10.1007/s13595-018-0793-8.

4. Adams, H.D.; MacAlady, A.K.; Breshears, D.D.; Allen, C.D.; Stephenson, N.L.; Saleska, S.R.; Huxman, T.E.; McDowell, N.G. Climate-induced tree mortality: Earth system consequences. Eos (Washington. DC). 2010, 91, 153-154, doi:10.1029/2010EO170003.

5. Dietze, M.C.; Moorcroft, P.R. Tree mortality in the eastern and central United States: Patterns and drivers. Glob. Chang. Biol. 2011, 17, 3312-3326, doi:10.1111/j.1365-2486.2011.02477.x.

6. Pfeifer, E.M.; Hicke, J.A.; Meddens, A.J.H. Observations and modeling of aboveground tree carbon stocks and fluxes following a bark beetle outbreak in the western United States. Glob. Chang. Biol. 2011, 17, 339350, doi:10.1111/j.1365-2486.2010.02226.x.

7. Trumbore, S.; Brando, P.; Hartmann, H. Forest health and global change. Science 2015, 349, 814-818.

8. Runkle, J.R. Canopy tree turnover in old-growth mesic forests of eastern north America. Ecology 2000, 81, 554-567, doi:10.1890/0012-9658(2000)081[0554:CTTIOG]2.0.CO;2.

9. Waters, W.E. Life-table approach to analysis of insect impact. J. For. 1969, 67, 300-304.

10. Morse, B.W.; Kulman, H.M. Plantation white spruce mortality: estimates based on aerial photography and analysis using a life-table format. Can. J. For. Res. 1984, 14, 195-200, doi:10.1139/x84-038.

11. Wyckoff, P.H.; Clark, J.S. Predicting tree mortality from diameter growth: A comparison of maximum likelihood and Bayesian approaches. Can. J. For. Res. 2000, 30, 156-167, doi:10.1139/x99-198.

12. Woodall, C.W.; Grambsch, P.L.; Thomas, W. Applying survival analysis to a large-scale forest inventory for assessment of tree mortality in Minnesota. Ecol. Modell. 2005, 189, 199-208, doi:10.1016/j.ecolmodel.2005.04.011.

13. Hiroshima, T. Applying age-based mortality analysis to a natural forest stand in Japan. J. For. Res. 2014, 19, 379-387, doi:10.1007/s10310-013-0428-8

14. Rinn, F. Eine neue Bohrmethode zur Holzuntersuchung. Holz-Zentralblatt. 1989, 34, 529-530..

15. Wijenayake, P.R.; Hiroshima, T. Survival analysis of a natural forest stand under the effect of wind disturbances -A case study of the preserved forest in Northern Japan- (under review).

16. Cox, D. R.; Oakes, D. Analysis of Survival Data; Chapman and Hall: London, 1984.

17. Crowder, M.J.; Kimber, A.C.; Smith, R.L. Statistical Analysis of Reliability Data.; Chapman and Hall: London, 1994; Vol. 27;

18. Klein, J. P.; Moeschberger, M.L. Survival Analysis: Techniques for Censored and Truncated Data; Springer, Ed.; Springer, NewYork, 1997.

19. Kaplan, E.L.; Meier, P. Nonparametric Estimation from Incomplete Observations. J. Am. Stat. Assoc. 1958, 53, 457-481, doi:10.1080/01621459.1958.10501452.

(C) 2020 by the authors. Licensee MDPI, Basel, Switzerland. This article is an open access article distributed under the terms and conditions of the Creative Commons Attribution (CC BY) license (http://creativecommons.org/licenses/by/4.0/). 